\title{
Atitudes frente a aspectos relevantes da prática médica: estudo transversal randomizado com alunos de segundo e sexto anos
}

PALAVRAS-CHAVE

- Atitude.

- Atitude frente a morte.

- Educação médica.

- Estudante de medicina.

- Saúde mental.

KEY WORDS

- Attitudes.

- Attitude to death.

- Education Medical.

- Students Medical.

- Mental health.

Recebido em: 18/10/2007

Reencaminhado em: 24/12/2007 Reencaminhado em 20/03/2008 Aprovado em: 10/04/2008

$40 \frac{\text { REVISTA BRASILEIRA DE EDUCAÇÃO MÉDICA }}{33(1): 40-48 ; 2009}$

\section{Attitudes toward relevant aspects of medical practice: a cross-sectional study with a random sample of second- and sixth-year students}

Adriana Rainha Mascia ${ }^{\mathrm{I}}$ Fernanda Braga Silva ${ }^{\mathrm{I}}$ Ana Cecilia Lucchese ${ }^{\mathrm{I}}$

Mario Alfredo De Marco ${ }^{\mathrm{I}}$ Maria Cezira Fantini Nogueira Martins ${ }^{\text {II }}$ Luiz Antonio Nogueira Martins ${ }^{\mathrm{I}}$

\section{R E S U M O}

Estudo transversal com duas amostras randomizadas de 50 alunos do segundo e 50 do sexto ano de graduação em Medicina. Foi aplicado um questionário com questões abertas e a escala "Instrumento de avaliação de atitudes de estudantes de medicina frente a aspectos relevantes da prática médica" (Colares, 2002). A escala contém 52 questões referentes à: 1. Aspectos psicológicos e emocionais nas doenças orgânicas e mentais; 2. Manejo de situações relacionadas à morte; 3. Atenção primária à saúde; 4 . Aspectos relacionados à doença mental; 5 . Contribuição do médico ao avanço científico da medicina; 6 . Outros aspectos relacionados à atuação médica è̀s políticas de saúde. As atitudes são categorizadas em positivas, negativas e conflitantes. Observou-se que os estudantes apresentaram atitudes positivas frente a pelo menos três dos seis aspectos abordados; os alunos do segundo ano e do sexto ano apresentaram diferença estatisticamente significativa (chi $\left.{ }^{2}=6,901, g . l .=1, p<0,05\right)$ nas atitudes relacionadas ao fator 2 (manejo de situações relacionadas à morte).

\section{A B S T R A C T}

This was a cross-sectional study with two random samples: 50 second-year and 50 sixth-year undergraduate medical students. An open-ended questionnaire was applied, in addition to a scale known as the "Instrument for evaluating medical students' attitudes towards key aspects of medical practice" (Colares, 2002). The scale contains 52 questions on: 1. Psychological and emotional aspects of physical and mental illness; 2 . managing situations related to death; 3. primary healthcare; 4 . aspects related to mental illness; 5 . the physician's contribution to scientific progress in medicine; and 6. other aspects of medical work and health policies. The attitudes are categorized as positive, negative, or conflictive. According to the findings, students had positive attitudes towards at least three of the six aspects. Second-year and sixth-year students differed significantly (chi ${ }^{2}=6.901$, d.f. $\left.=1, p<0.05\right)$ in their attitudes toward factor 2 (managing situations related to death).

\footnotetext{
I Universidade Federal de São Paulo, São Paulo, Brasil.

${ }^{\text {II }}$ Secretaria de Estado da Saúde de São Paulo, Instituto de Saúde, São Paulo, Brasil.
} 


\section{INTRODUÇÃO}

As escolas médicas têm o objetivo de formar não só bons profissionais do ponto de vista técnico, mas, também, indivíduos capazes de desenvolver atitudes construtivas e adaptadas ao meio social de determinada realidade populacional ${ }^{1}$. De maneira geral, espera-se que o médico, ao receber formação adequada, possa desenvolver, em sua prática assistencial, atitudes profissionais que contemplem os desejos tanto de curar como de cuidar ${ }^{2-3}$.

A graduação em Medicina tende a exigir do aluno um complexo preparo técnico-relacional, desenvolvido a partir de estudos teóricos e experiências assistenciais supervisionadas. Lidar com pessoas doentes, com as reações emocionais dos pacientes e familiares, conviver com a morte, administrar as vivências de impotência frente às dificuldades e limitações do conhecimento médico e dos sistemas de saúde são algumas das múltiplas situações experimentadas pelos estudantes durante o curso de Medicina. Estas experiências têm impacto na forma como os estudantes passam a perceber o exercício profissional e nas intenções ligadas a como pretendem desempenhá-lo no futuro ${ }^{4-8}$.

Estudos mostram que, durante a graduação em Medicina, simultaneamente ao desenvolvimento de competências e habilidades, há um incremento do ceticismo e uma diminuição do idealismo e da capacidade empática ${ }^{9-11}$. Levando-se em conta que as atitudes podem ser consideradas fortes preditores de comportamentos e ações diante de determinado objeto e em relação a ele, conhecer as atitudes dos estudantes frente a situações relevantes da prática médica e a influência da graduação médica sobre essas atitudes pode contribuir para aperfeiçoar a formação desses futuros profissionais ${ }^{12-13}$.

\section{OBJETIVO}

Investigar as atitudes dos alunos da graduação médica frente a situações relevantes da prática médica e comparar atitudes de alunos do segundo e do sexto ano. Analisar se as atitudes diferem em função de variáveis sociodemográfico-acadêmicas e da futura especialidade médica pretendida.

\section{MÉTODOS}

O projeto de pesquisa foi previamente aprovado pelo Comitê de Ética em Pesquisa da instituição.

A pequisa foi desenhada como estudo transversal randomizado com 50 alunos do segundo ano e 50 alunos do sexto ano do curso de graduação em Medicina. Foram sorteados 50 alunos do conjunto de estudantes de cada um dos anos selecionados para o estudo (cada ano contava com um total de alunos entre 100 e 120). Ter sido sorteado e ser aluno do segundo e do sexto anos fo- ram os critérios para inclusão no estudo. Aos alunos sorteados foi entregue em novembro de 2005 um caderno que continha:

1. termo de consentimento livre e esclarecido;

2. escala psicométrica intitulada "Instrumento para avaliação de atitudes de estudantes de medicina frente a aspectos relevantes da prática médica", desenvolvida e validada por Colares et al. ${ }^{12}$;

3. questionário sociodemográfico-acadêmico desenvolvido para este estudo;

4. três questões abertas com a seguinte redação:

a) descreva suas experiências associadas às situações de morte;

b) relate experiências gratificantes e estressantes vivenciadas durante a graduação;

c) espaço para comentários e reflexões a respeito das experiências como estudante de Medicina.

A escala "Instrumento para avaliação de atitudes de estudantes de medicina frente a aspectos relevantes da prática médica" é composta por 52 itens e avalia seis fatores:

\section{Fator 1}

Aspectos psicológicos e emocionais em doenças orgânicas e mentais. Este fator, composto por 11 questões, visa avaliar o grau de importância que os alunos atribuem ao papel dos estados emocionais no curso de uma doença;

\section{Fator 2}

Manejo de situações relacionadas à morte. $\mathrm{O}$ objetivo deste fator, que tem oito questões, é conhecer o grau de dificuldade dos alunos diante de situações associadas à morte e ao morrer (exemplo: vivências diante da morte; ter que dar a notícia a uma família);

\section{Fator 3}

Atenção primária à saúde. Este fator é constituído por 11 afirmações sobre a importância do médico generalista e da prática da prevenção por qualquer especialista médico;

\section{Fator 4}

Aspectos relacionados à doença mental. Este fator analisa, por meio de oito afirmações, os sentimentos do estudante diante do doente mental e as dificuldades enfrentadas;

\section{Fator 5}

Contribuição do médico ao avanço científico da medicina. $\mathrm{O}$ objetivo das seis afirmações deste item é saber o que os alunos pensam sobre a importância da pesquisa na carreira médica; 


\section{Fator 6}

Outros aspectos relacionados à atuação médica e às políticas de saúde. Por meio de oito questões, este fator analisa as atitudes dos estudantes diante de aspectos diversos, referentes à atuação médica, tanto em serviços de atenção primária à saúde, como em outros de maior complexidade. Focaliza, também, algumas questões que envolvem as políticas de saúde, especificamente na área de saúde mental.

Para cada item, havia cinco opções de respostas: 1 - estou totalmente de acordo; 2 - concordo em parte; 3 - estou em dúvida; 4 -discordo em parte; 5 - estou totalmente em desacordo. As atitudes são classificadas em três categorias, conforme a pontuação das médias (M): $\mathrm{M}<3$ : atitudes predominantemente negativas;
M entre 3 e 3,9: atitudes conflitantes ou indefinidas; $M>4$ : atitudes predominantemente positivas.

Os dados foram analisados pelo teste de $\mathbf{X}^{2}$, empregando-se $\mathrm{o}$ programa SPSS (Statistical Package for Social Sciences).

\section{RESULTADOS}

A amostra final foi composta por 82 alunos, 41 do sexto ano e 41 do segundo, sendo $55,6 \%$ do sexo masculino e $44,4 \%$ do sexo feminino. A média de idade foi de 23,2 anos, 54,9\% eram procedentes da Grande São Paulo e 60,5\% moravam com familiares. Cinquenta e cinco por cento dos alunos pretendiam se especializar em áreas clínicas e 37,5\% em especialidades cirúrgicas; o restante pretendia outras áreas, tais como pesquisa (Tabela 1).

Tabela 1

Descrição dos dados sociodemográficos da amostra

\begin{tabular}{lccc}
\hline & Segundo ano & Sexto ano & Total \\
\hline Proporção sexo masculino & $17(41,5 \%)$ & $29(70,7 \%)$ & $46(56,1 \%)$ \\
Proporção sexo feminino & $24(51,5 \%)$ & $12(29,3 \%)$ & $36(43,9 \%)$ \\
Pretendem a especialidade clínica & & & \\
Pretendem a especialidade cirúrgica & $20(51,3 \%)$ & $24(58,5 \%)$ & $44(55,0 \%)$ \\
Outras & $16(41,0 \%)$ & $14(34,1 \%)$ & $30(37,5 \%)$ \\
& $3(7,7 \%)$ & $3(7,3 \%)$ & $6(7,5 \%)$ \\
Procedente da Grande São Paulo & & & $45(54,9 \%)$ \\
Procedente do interior de SP & $19(46,3 \%)$ & $26(63,4 \%)$ & $32(39,0 \%)$ \\
Outros & $18(43,9)$ & $14(34,1 \%)$ & $5(6,1 \%)$ \\
& $4(9,8)$ & $1(2,4 \%)$ & $49(60,5 \%)$ \\
Moram com familiares & & & $24(29,6 \%)$ \\
Moram com amigos & $22(53,7 \%)$ & $8(20,0 \%)$ & $8(9,9 \%)$ \\
Outros & $16(39,0 \%)$ & $6(12,5 \%)$ & $16(19,5 \%)$ \\
Possui pais médicos & $3(7,3 \%)$ & $10(24,4 \%)$ & $66(80,5 \%)$ \\
Não possui pais médicos & & $31(75,6 \%)$ & \\
\hline
\end{tabular}

Tivemos 18\% de perdas ( $\mathrm{n}=9$ ) em cada ano da graduação, e não houve diferença estatística entre as perdas e a amostra final em relação aos dados sociodemográficos. 
Escala de atitudes

Nos dois grupos - segundo e sexto anos -, foram observadas: atitudes positivas frente a pelo menos três dos seis fatores avaliados; atitudes diferentes estatisticamente frente ao fator 2 (manejo de situações relacionadas à morte) e atitudes sem diferença significativa em relação aos demais fatores (Tabela 2).
Os alunos do segundo ano apresentaram maior percentual de atitudes predominantemente positivas frente aos fatores 1,3 , 5 e 6 , e maior percentual de atitudes conflitantes ou predominantemente negativas frente aos outros fatores (Tabela 3).

Tabela 2

Classificação das atitudes do total de alunos frente a aspectos relevantes da prática médica

Desempenho dos alunos

\begin{tabular}{|c|c|c|c|}
\hline \multirow[b]{2}{*}{ Fator } & \multicolumn{3}{|c|}{ Atitude } \\
\hline & $\begin{array}{c}\text { Predominantemente } \\
\text { Positiva }\end{array}$ & Conflitante & $\begin{array}{c}\text { Predominantemente } \\
\text { Negativa }\end{array}$ \\
\hline (1) Aspectos emocionais em doenças orgânicas & $60(79,0 \%)$ & $14(18,4 \%)$ & $2(2,6 \%)$ \\
\hline (2) Situações relacionadas à morte & $10(12,8 \%)$ & $33(42,3 \%)$ & $35(44,9 \%)$ \\
\hline (3) Atenção primária à saúde & $54(69,2 \%)$ & $19(24,4 \%)$ & $5(6,4 \%)$ \\
\hline (4) Aspectos relacionados à doença mental & $18(23,3 \%)$ & $29(37,7 \%)$ & $30(39,0 \%)$ \\
\hline (5) Contribuição do médico ao avanço científico da Medicina & $42(52,5 \%)$ & $27(33,8 \%)$ & $11(13,7 \%)$ \\
\hline (6) Outros aspectos da atuação médica na comunidade & $65(83,3 \%)$ & $12(15,4 \%)$ & $1(1,3 \%)$ \\
\hline
\end{tabular}

Tabela 3

Classificação das atitudes dos alunos do segundo ano frente a aspectos relevantes da prática médica

Desempenho dos alunos do segundo ano

Atitude

Fator

\begin{tabular}{ccc}
\hline & Atitude \\
\hline $\begin{array}{c}\text { Predominantemente } \\
\text { Positiva }\end{array}$ & Conflitante & $\begin{array}{c}\text { Predominantemente } \\
\text { Negativa }\end{array}$ \\
\hline $34(89,5 \%)$ & $4(10,5 \%)$ & 0 \\
$4(10,5 \%)$ & $11(29,0 \%)$ & $23(60,5 \%)$ \\
$25(65,8 \%)$ & $10(26,3 \%)$ & $3(7,9 \%)$ \\
$8(20,5 \%)$ & $17(43,6 \%)$ & $14(35,9 \%)$ \\
$26(65,0 \%)$ & $9(22,5 \%)$ & $5(12,5 \%)$ \\
$32(80,0 \%)$ & $7(17,5 \%)$ & $1(2,5 \%)$ \\
\hline
\end{tabular}


Os alunos do sexto ano apresentaram maior percentual de atitudes predominantemente positivas frente aos fatores 1, 3 e 6 , e maior percentual de atitudes conflitantes ou predominantemente negativas frente aos outros (Tabela 4).

Tabela 4

Classificação das atitudes dos alunos do sexto ano frente a aspectos relevantes da prática médica

Desempenho dos alunos do sexto ano

\begin{tabular}{|c|c|c|c|}
\hline \multirow[b]{2}{*}{ Fator } & \multicolumn{3}{|c|}{ Atitude } \\
\hline & $\begin{array}{c}\text { Predominantemente } \\
\text { Positiva }\end{array}$ & Conflitante & $\begin{array}{c}\text { Predominantemente } \\
\text { Negativa }\end{array}$ \\
\hline (1) Aspectos emocionais em doenças orgânicas & $26(68,4 \%)$ & $10(26,3 \%)$ & $2(5,3 \%)$ \\
\hline (3) Atenção primária à saúde & $29(72,5 \%)$ & $9(22,5 \%)$ & $2(5,0 \%)$ \\
\hline (4) Aspectos relacionados à doença mental & $10(26,3 \%)$ & $12(31,6 \%)$ & $16(42,1 \%)$ \\
\hline (5) Contribuição do médico ao avanço científico da Medicina & $16(40,0 \%)$ & $18(45,0 \%)$ & $6(15,0 \%)$ \\
\hline
\end{tabular}

Oresultado de cada fator foi comparado entre os anos, e o único fator que apresentou diferença estatisticamente significativa foi o fator 2 (situações relacionadas à morte), em que a maioria dos alu- nos do segundo ano apresentou atitude predominantemente negativa, e do sexto ano, atitude conflitante (ora positiva, ora negativa)

(Tabela 5).

Tabela 5

Proporção da classificação feita por alunos do segundo e sexto anos das questões do fator 2

\begin{tabular}{|c|c|c|c|}
\hline Atitude predominantemente positiva & $4(10,5 \%)$ & $6(15,0 \%)$ & $10(12,8 \%)$ \\
\hline Atitude conflitante & $11(28,9 \%)$ & $22(55,0 \%)$ & $33(42,3 \%)$ \\
\hline Atitude predominantemente negativa & $23(60,6 \%)$ & $12(30,0 \%)$ & $35(44,9 \%)$ \\
\hline
\end{tabular}

Teste qui-quadrado $=6,901$, g.l $.=1, \mathrm{p}<0,05$ 


\section{Questões abertas}

Os principais resultados obtidos nas questões abertas foram os seguintes:

\section{Experiências com a morte}

A maioria dos alunos, tanto do segundo como do sexto ano, que tiveram experiências relacionadas com a morte referiram que estas experiências foram associadas à morte de algum parente ou pessoa próxima; poucos alunos referiram ter tido experiências relacionadas com a morte de pacientes durante o curso de graduação.

Os alunos do sexto ano falaram sobre aprendizado e aceitação da morte, enquanto os alunos do segundo ano ressaltaram o lado negativo da experiência, utilizando palavras como: "culpa", "raiva", "sentimento ruim".

\section{Aspectos gratificantes durante a graduação}

A grande maioria dos alunos salientou que a relação com os pacientes é o maior motivo de gratificação durante a graduação, especialmente nos momentos em que estes manifestam seu agradecimento e reconhecimento pelo atendimento realizado.

\section{DISCUSSÃO}

A semelhança entre os grupos no que diz respeito ao predomínio de atitudes positivas frente aos fatores 1 (Aspectos psicológicos e emocionais nas doenças orgânicas), 3 (Atenção primária à saúde) e 6 (Outros aspectos da atuação médica) sugere que, aparentemente, essas atitudes positivas são intrínsecas ao aluno, provavelmente relacionadas com aspectos vocacionais, conforme salienta Millan ${ }^{14}$ em seu estudo sobre "vocação médica". Assim, uma provável hipótese para esta homogeneidade de atitude estaria relacionada com características pessoais dos alunos que escolhem a medicina como profissão. Eles estariam predispostos, de certa forma, a agir adequadamente diante dessas situações complexas de sofrimento e doença, a desenvolver funções sociais relacionadas ao cuidar de pessoas doentes.

A diferença nas atitudes frente ao fator 2 (Manejo de situações relacionadas à morte), com os alunos do segundo ano apresentando maior percentual de atitudes predominantemente negativas, e os do sexto, atitudes conflitantes (ora positivas, ora negativas), aponta para a hipótese de que, no decorrer do curso, o aluno teria algumas experiências que favorecem a reflexão e um posicionamento mais amadurecido diante das situações envolvidas com a morte.

Entretanto, o baixo escore nesse fator, ou seja, a prevalência de atitudes mais conflitantes e negativas, é um indicativo da complexidade que o tema morte representa para os alunos. Esse tema desperta, nos seres humanos em geral, sentimentos variados, sendo a maioria deles negativos e desconfortáveis ${ }^{5,15}$. Na prática médica, tem sido observado que o profissional que não está devidamente preparado para lidar com essas situações poderá experimentar uma gama de sentimentos reativos, como: solidão profissional, perda do senso de missão, cinismo, desesperança, frustração, bem como risco aumentado de burnout e depressão. E esses sentimentos reativos podem ter repercussões negativas no cuidado ao paciente e à família ${ }^{16}$.

Esses dados indicam claramente que não podemos subestimar a importância do preparo do futuro médico nessa área. Nosso desafio é como favorecer esse preparo de uma forma efetiva e eficiente de modo a proporcionar uma reflexão e um desenvolvimento das habilidades para lidar com esse campo tão carregado emocionalmente.

Isso pode ser feito, por exemplo, por meio de cursos sobre tanatologia que abordem temas como cuidados paliativos, cuidados ao paciente terminal e à sua família, e por ações mais gerais que introduzam o tema nos diferentes cenários de aprendizado e atendimento. Oestímulo ao assunto durante as discussões de casos clínicos e a criação de grupos de apoio, tanto para as famílias quanto para os profissionais, são medidas favorecedoras do autocuidado (para alunos e profissionais) e do cuidado com o outro (para pacientes e famílias). Em algumas universidades, numa perspectiva integradora, os cursos de psicologia médica contemplam o tema e promovem atividades em que a abordagem é realizada conjuntamente pelos professores de psicologia médica e professores das diferentes especialidades ${ }^{2,17}$.

Nas respostas às questões abertas desta pesquisa, observamos que a vivência maior que os estudantes possuem sobre esse assunto está relacionada às experiências pessoais, como morte de familiares ou amigos, ou seja, experiências não relacionadas com o curso de graduação, e por isso, na maioria das vezes, sem conteúdo reflexivo. Este pode ser também um motivo pelo qual estudantes com experiências de perda e luto têm maior dificuldade em lidar com pacientes terminais e seus familiares ${ }^{1,5,15}$. A dificuldade pode estar relacionada a lembranças de experiências vividas no passado distante e que são reativadas em situações assistenciais atuais, como podemos observar no caso de uma aluna do sexto ano (24 anos, procedente da Grande São Paulo, sem pais médicos e que pretendia a área cirúrgica) que escreveu o seguinte na questão aberta sobre experiências com a morte:

[...]uma paciente japonesa e idosa que chegou com parada na emergência clínica, quando eu passava por lá, no sexto ano. Ela tinha câncer de mama terminal, com várias metástases em pele e gradeado costal e, no lugar dos seios, havia apenas úlceras horríveis. Tentaram reanimá-la em vão. Lembrei de minha avó (também japonesa), que faleceu de câncer. Penso se ela também sofreu com essas horríveis feridas, pois eu não me lembro, era criança... Ela era extremamente vaidosa. 
Outro fator que pode contribuir para que os alunos tenham dificuldade em apresentar atitudes mais positivas com relação ao manejo de situações relacionadas à morte é aquilo que alguns autores denominam "conspiração do silêncio"18, ou seja, uma evitação desses temas por parte dos estudantes e professores, havendo às vezes certa tendência a censurar aqueles que demonstram seus sentimentos, interpretados como sinal de fraqueza psicológica. Essa conspiração, ao inibir manifestações de emoções e sentimentos, certamente dificulta a discussão sobre temas relacionados à morte. Assim, apesar de a morte ser um dos fatores mais estressantes e impactantes durante a graduação ${ }^{5}$, a procura ativa de conhecimento e discussão dos estudantes e médicos sobre o assunto é escassa.

Outro fator correlacionado diz respeito ao ambiente altamente competitivo que predomina nas escolas médicas, o que contribui para a manutenção da "conspiração do silêncio" e para o evitamento de trocas afetivas ou intimidades. Considerando-se a conhecida tendência de uma parte dos alunos de Medicina a apresentar um perfil caracterizado como "pessoas brilhantes, individualistas, altamente competitivas e que não se mostram inclinadas a compartilhar sentimentos e sensações", torna-se importante enfatizar a necessidade de criar um ambiente de aprendizagem favorecedor do intercâmbio afetivo-relacional. Não é surpreendente, portanto, o fato de que os estudantes de Medicina mostrem dificuldade em estabelecer vínculos estreitos com seus colegas e, por vezes, sintam-se solitários e com sintomas depressivos ${ }^{4,5}$. Uma aluna do sexto ano (27 anos, procedente de outro estado que não São Paulo, sem pais médicos e que pretendia a área clínica) se refere a alguns destes temas em seu relato sobre suas experiências como estudante de Medicina:

Foi muito diferente (pior e melhor em vários aspectos) do que eu imaginava. Continuo a acreditar nos meus valores, mas desacreditei na possibilidade de mudança. Aprendi a não confiar em ninguém (de cara ou com pouco tempo de convivência), a não esperar nada de bom dos outros, a contar só comigo.

Aspectos positivos relacionados ao crescimento que o curso pode proporcionar foram salientados por uma aluna do sexto ano (26 anos, procedente da Grande São Paulo, sem pais médicos e que pretendia a área clínica) ao descrever suas experiências na graduação sobre o tema morte:

As experiências (diante da morte) são várias durante o curso médico. O que muda durante os anos é a maneira como recebemos o fato da mor- te. No início do curso, achava que o melhor, para o paciente, seria que fosse evitada a sua morte a qualquer custo, não importando o que precisasse ser feito. E eu sentia uma angústia frente aos pacientes que aceitavam a sua morte antes mesmo de tentar as últimas medidas. Hoje em dia, continuo me sentindo impotente frente a alguns tipos de pacientes, só que agora compreendo que os pacientes têm o direito de decidir sobre o seu tratamento juntamente com a equipe médica. Continuo achando que todas as formas de tratamento devem ser oferecidas e esclarecidas aos pacientes, mas não necessariamente postas em prática.

Apesar das dificuldades dos alunos frente a situações que envolvem a morte, há trabalhos que demonstram que de $30 \%$ a $60 \%$ dos médicos aprendem a lidar de forma adequada com pacientes terminais durante a residência médica graças ao amadurecimento pessoal e ao maior contato com o paciente ${ }^{15}$.

No que diz respeito às atitudes dos estudantes frente à doença mental (fator 4), em especial se considerarmos a epidemiologia das doenças mentais, a atitude predominantemente negativa se revela preocupante. Segundo a Organização Mundial de Saúde, cinco das dez causas-líderes de incapacidade atualmente são distúrbios psiquiátricos como depressão unipolar, abuso de substâncias, transtorno bipolar, esquizofrenia e transtorno obsessivo-compulsivo. Se considerarmos ainda que $57,4 \%$ dos pacientes internados em hospitais gerais apresentam algum sintoma psiquiátrico, como depressão, ansiedade e/ou quadro confusional $^{19}$, o resultado desse fator adquire maior importância ainda. Além disso, é relevante considerarmos, no que tange ao tema doença mental, a questão da saúde mental dos próprios estudantes; alguns estudos mostram que a prevalência de depressão e ansiedade entre os estudantes de Medicina é maior do que na população geral ${ }^{20}$.

Um estudo sobre saúde mental realizado com médicos que trabalham em cuidados primários de saúde no sul do Brasil ${ }^{21}$ constatou que médicos clínicos têm muita dificuldade em diagnosticar e tratar os problemas mentais e que estas dificuldades estão associadas a vários fatores, como a necessidade do envolvimento dos familiares nos cuidados, a baixa adesão ao tratamento prolongado e a dificuldade em lidar com sintomas psicossomáticos.

Deve-se levar em consideração, também, que o preconceito e atitudes estereotipadas diante do doente mental são frequentes na população em geral, o que provavelmente contribui para o baixo escore no fator e valoriza a necessidade de intervenções 
educacionais específicas durante a graduação, com a finalidade de modificar e melhorar esse contexto ${ }^{17}$. Estas intervenções devem prever a oferta de maior contato dos estudantes com pacientes psiquiátricos e seus familiares e o conhecimento dos múltiplos recursos terapêuticos disponíveis atualmente.

O predomínio de atitudes positivas dos alunos de segundo ano e conflitantes do sexto ano em relação ao fator 5 (Contribuição do médico no avanço científico da medicina) pode ser consequência do maior interesse pelos projetos de iniciação científica durante o começo do curso, quando os alunos têm maior disponibilidade de tempo, e da maior dificuldade dos internos para se dedicar à pesquisa, atarefados com o cotidiano do internato e preocupados com o exame de seleção para a residência médica. Outro fator que talvez também contribua para esse processo é a presença de professores antimodelos ${ }^{7}$ - excelentes pesquisadores que não têm habilidades didáticas e/ou clínicas e que não tendem a estabelecer relações empáticas com os pacientes e com os alunos-, que estão presentes em todas as faculdades e costumam ser reconhecidos e relembrados pelos alunos e colegas como aqueles a quem não encaminharíamos nossos parentes e amigos.

Vale salientar que os resultados encontrados em nosso estudo foram, em parte, semelhantes aos obtidos no estudo pioneiro de Trocon et al. ${ }^{13}$, que avaliaram as atitudes de graduandos em Medicina em relação a aspectos relevantes da prática médica durante quatro anos consecutivos numa escola médica do interior de São Paulo. Comparando-se os resultados destes estudos, notam-se as seguintes semelhanças e diferenças:

- Semelhanças quanto ao predomínio de atitudes positivas em relação aos fatores 1 (Aspectos psicológicos e emocionais em doenças orgânicas e mentais), 3 (Atenção primária à saúde) e 6 (Outros aspectos relacionados à atuação médica e às políticas de saúde);

- Diferenças quanto aos demais fatores: os estudantes da citada pesquisa revelaram atitudes conflitantes, enquanto nosso estudo revelou o predomínio de atitudes positivas diante do fator 5 (Contribuição do médico ao avanço científico da medicina) e atitudes predominantemente negativas frente aos fatores 2 (Manejo de situações relacionadas à morte) e 4 (Aspectos relacionados à doença mental);

- Comparando-se as atitudes dos alunos do sexto ano dos dois estudos, nota-se que na pesquisa realizada por Troncon et $^{1 l^{13}}$. houve predomínio de atitudes conflitantes diante do fator 4 (Aspectos relacionados à doença mental), enquanto em nosso grupo as atitudes foram predominantemente negativas.

Para finalizar, estudos indicam que, no começo do curso, os alunos tendem a se identificar mais com o paciente e, com o transcorrer do curso, passam a se identificar mais com o médi$\mathrm{co}^{6}$. Essa identificação inicial é importante, pois tende a se refletir numa prática assistencial em que o aluno se sensibiliza com a condição do paciente e procura escolher as melhores opções de conduta e tratamento, ou seja, desenvolve a capacidade empáti$\mathrm{ca}^{22}$. Na passagem para a posição de médico, essa sensibilidade pode se modificar - com predomínio de mecanismos psicológicos de autoproteção (defesas do ego, como negação, dissociação e intelectualização) -, e o profissional pode desenvolver comportamentos rígidos e estereotipados, tornando-se por vezes negativista, arrogante e com perda da capacidade de se compadecer com o sofrimento dos outros ${ }^{22}$, como mostram alguns estudos que sugerem que alguns jovens idealistas, ingressantes no curso, se transformam em profissionais frios e distantes ${ }^{23}$.

Nossos resultados contribuem para salientar que as dificuldades diante de situações que envolvem morte e doença mental merecem maior destaque na graduação. Algumas estratégias devem ser implementadas, como ênfase no treinamento em Saúde Mental e Cuidados Paliativos e na criação de serviços de assistência psicológica e psiquiátrica aos estudantes, como já ocorre em várias universidades do País ${ }^{3,4}$.

Formar médicos tecnicamente bem preparados, éticos, com capacidade de desenvolver relações empáticas com os pacientes e com responsabilidade social é uma das missões da escola médica. Para isso, o processo educacional deve estar atento para uma formação, com caráter biopsicossocial, e para o desenvolvimento de habilidades interpessoais ${ }^{24}$.

\section{REFERÊNCIAS}

1. Lima VV. Avaliação de competências nos cursos médicos In: Marins JJN, Rego S, Lampert JB, Araújo JGC (Org.). Educação médica em transformação. Rio de Janeiro; São Paulo: Abem; Hucitec; 2004.

2. De Marco MA. A psicologia médica na EPM-UNIFESP. In: De Marco MA (org) A Face Humana da Medicina. São Paulo: Casa do Psicólogo; 2003.

3. Nogueira-Martins LA. Residência médica: estresse e crescimento. São Paulo: Casa do Psicólogo, 2005

4. Millan LR, De Marco OLN, Rossi E, Arruda PCV. O universo psicológico do futuro médico. São Paulo: Casa do Psicólogo; 1999.

5. Silva LCG. Eventos estressantes na relação com o paciente e estratégias de enfrentamento: estudo com acadêmicos de medicina. J Bras Psiquiatr. 2004;53(3):185-196.

6. Ramalho JF. Identidade médica. In: De Marco MA (Org.). A face humana da medicina. São Paulo: Casa do Psicólogo; 2003. 
7. Nogueira-Martins MCF, Nogueira-Martins LA, Turato ER. Medical students' perceptions of their learning about the physician-patient relationship: a qualitative study. Med Educ. 2006;40:322-328.

8. Ramos-Cerqueira ATA, Lima MC. A formação da identidade do médico: implicações para o ensino de graduação em medicina. Interface (Botucatu). 2002;6:107-116.

9. Hojat M, Mangione S, Nasca TJ et al. An empirical study of decline in empathy in medical school. Med Educ. 2004;38(9):934-941.

10. Spencer J. Decline in empathy in medical education: how can we stop the rot? Med Educ. 2004;38(9):916-918.

11. Woloschuk W, Harasym PH, Temple W. Attitude change during medical school: a cohort study. Med Educ. 2004;38:522-34.

12. Colares MFA, Troncon LEA, Figueiredo JFC. Construção de um instrumento para avaliação das atitudes de estudantes de medicina frente a aspectos relevantes da prática médica. Rev Bras Educ Med. 2002;26(3):194-203.

13. Troncon LEA, Colares MFA, Figueiredo JFC. Atitudes de graduandos em medicina em relação a aspectos relevantes da prática médica. Rev Bras Educ Med. 2003;27:20-28.

14. Millan LR. Vocação médica: um estudo de gênero. São Paulo: Casa do Psicólogo; 2005.

15. Vianna A, Piccelli H. O estudante, o médico e o professor de medicina perante a morte e o paciente terminal. Rev Ass Med Brasil. 1998;44(1):21-27.

16. Meier DE, Back AL, Morrison S. The inner life of physicians and care of the seriously iII. JAMA. 2001;286(23):3007-3014.

17. De Marco MA, Lucchese AC, Dias CC. Psicologia médica e semiologia integrada. In: Puccini RF (Org.). A formação médica na UNIFESP: excelência técnica e compromisso social. São Paulo: Ed. FAP. No prelo. 2008.
18. Avancine MATO, Jorge MR. Medos, atitudes e convicções de estudantes de medicina perante a doença. Psiq Prat Med. 2000; 33(1):2-9.

19. Lucchese AC, Citero VA, DeMarco MA, Andreoli SB, Nogueira-Martins LA. Relatives' needs of general hospital inpatients. São Paulo Med J. No prelo. 2008.

20. Dyrbye LN, Thomas Mr, Shanafelt TD. Systematic review of depression, anxiety, and other indicators of psychological distress among U.S. and Canadian medical students. Acad Med 2006;81(4):354-373.

21. Ballester DA, Filippon AP, Braga C, Andreoli SB. The general practitioner and mental health problems: challenges and strategies for medical education. São Paulo Med J. 2005;123(2):72-76.

22. Benbassat J, Baumal R. What is empathy, and how can it be promoted during clinical clerkships? Acad Med. 2004;79(9): 832-839.

23. Griffith $\mathrm{CH}$, Wilson JF. The loss of idealism throughout internship. Eval Health Prof. 2003; 26 (4):415-426.

24. Nogueira-Martins LA, Nogueira-Martins MCF. O exercício atual da medicina e a relação médico-paciente. Rev Bras Clin Terap. 1998; 24(2):59-64.

\section{CONFLITO DE INTERESSES}

Declarou não haver.

\section{ENDEREÇO PARA CORRESPONDÊNCIA}

Adriana Rainha Mascia

Rua Três de Maio, 154 - apto 142

Vila Clementino

CEP. 04044-020 - São Paulo - SP

E-mail: drimascia@yahoo.com.br 